\title{
Concentrated oat $\beta$-glucan, a fermentable fiber, lowers serum cholesterol in hypercholesterolemic adults in a randomized controlled trial
}

\author{
Katie M Queenan ${ }^{1}$, Maria L Stewart ${ }^{1}$, Kristen N Smith ${ }^{1}$, William Thomas ${ }^{2}$, R \\ Gary Fulcher ${ }^{1,3}$ and Joanne L Slavin*1
}

Address: ${ }^{1}$ Department of Food Science and Nutrition, University of Minnesota, St. Paul, MN, USA, ${ }^{2}$ Division of Biostatistics, School of Public Health, University of Minnesota, Minneapolis, MN, USA and ${ }^{3}$ Department of Food Science, University of Manitoba, Winnipeg, Manitoba, Canada

Email: Katie M Queenan - jslavin@umn.edu; Maria L Stewart - stew0257@umn.edu; Kristen N Smith - knsmith@umn.edu; William Thomas - thoma003@umn.edu; R Gary Fulcher - fulcher@cc.manitoba.ca; Joanne L Slavin* - jslavin@umn.edu

* Corresponding author

Published: 26 March 2007

Nutrition Journal 2007, 6:6 doi:10.1186/1475-2891-6-6

This article is available from: http://www.nutritionj.com/content/6/1/6

(C) 2007 Queenan et al; licensee BioMed Central Ltd.

This is an Open Access article distributed under the terms of the Creative Commons Attribution License (http://creativecommons.org/licenses/by/2.0), which permits unrestricted use, distribution, and reproduction in any medium, provided the original work is properly cited.

\begin{abstract}
Background: Soluble fibers lower serum lipids, but are difficult to incorporate into products acceptable to consumers. We investigated the physiological effects of a concentrated oat $\beta$-glucan on cardiovascular disease (CVD) endpoints in human subjects. We also compared the fermentability of concentrated oat $\beta$-glucan with inulin and guar gum in a model intestinal fermentation system.
\end{abstract}

Methods: Seventy-five hypercholesterolemic men and women were randomly assigned to one of two treatments: 6 grams/day concentrated oat $\beta$-glucan or 6 grams/day dextrose (control). Fasting blood samples were collected at baseline, week 3 , and week 6 and analyzed for total cholesterol, HDL cholesterol, LDL cholesterol, triglycerides, glucose, insulin, homocysteine and C-reactive protein (CRP). To estimate colonic fermentability, $0.5 \mathrm{~g}$ concentrated oat $\beta$-glucan was incubated in a batch model intestinal fermentation system, using human fecal inoculum to provide representative microflora. Fecal donors were not involved with the $\beta$-glucan feeding trial. Inulin and guar gum were also incubated in separate serum bottles for comparison.

Results: Oat $\beta$-glucan produced significant reduction from baseline in total cholesterol $(-0.3 \pm 0.1$ $\mathrm{mmol} / \mathrm{L})$ and $\mathrm{LDL}$ cholesterol $(-0.3 \pm 0.1 \mathrm{mmol} / \mathrm{L})$, and the reduction in $\mathrm{LDL}$ cholesterol were significantly greater than in the control group $(p=0.03)$. Concentrated oat $\beta$-glucan was a fermentable fiber and produced total SCFA and acetate concentrations similar to inulin and guar gum. Concentrated oat $\beta$-glucan produced the highest concentrations of butyrate at 4,8 , and 12 hours.

Conclusion: Six grams concentrated oat $\beta$-glucan per day for six weeks significantly reduced total and LDL cholesterol in subjects with elevated cholesterol, and the LDL cholesterol reduction was greater than the change in the control group. Based on a model intestinal fermentation, this oat $\beta$ glucan was fermentable, producing higher amounts of butyrate than other fibers. Thus, a practical dose of $\beta$-glucan can significantly lower serum lipids in a high-risk population and may improve colon health. 


\section{Background}

Cardiovascular disease (CVD) remains the top cause of death in the United States, with saturated fat and trans fat intake, serum cholesterol, and obesity as major risk factors. Diets recommended for improvement of cardiovascular risk factors include a diet high in dietary fiber. Cereal fibers that are high in water-soluble fiber, such as $\beta$-glucan, may improve cardiovascular disease risk through improvements in serum cholesterol and other intermediary risk factors. Oats are high in soluble fiber and appear to reduce CVD risk when consumed as part of a moderate fat, balanced diet. Soluble fiber $\beta$-glucan is thought to be the active component for the cholesterol lowering effect of oats. Few studies have been conducted on the effectiveness of concentrated $\beta$-glucan from oats and changes in physiological endpoints.

Concentrated $\beta$-glucan from oat is now commercially available as a dietary supplement. Oat $\beta$-glucan is a natural polymer comprised of individual glucose molecules that are linked together by a series of $\beta-(1-3)$ and $\beta-(1-4)$ linkages. Beta-glucan was identified as the major fermentable component in both cooked and uncooked rolled oats; the beta-glucan was not affected by cooking [1]. Rolled oats and isolated beta-glucan (termed oat gum in the report) contributed to increased gut viscosity in male Wistar rats [2].

The hypothesis that soluble dietary fiber lowers blood cholesterol levels by interfering with the absorption of dietary cholesterol is appealing. Increased gut viscosity may prevent dietary cholesterol from reaching the intestinal epithelium. However, it is unlikely that fiber-induced hypocholesterolemia is solely a result of this mechanism [3]. Exogenenous cholesterol represents only about one quarter of the body's cholesterol, and a significant change in absorption would lead to even smaller changes in blood cholesterol levels. Some fibers may decrease absorption of dietary cholesterol by altering the composition of the bile acid pool. Pectin and oat bran increased the portion of the total bile acid pool that was deoxycholic acid (DCA) [4]. DCA has been noted to decrease the absorption of exogenous cholesterol in humans [5]. Fermentation products may also alter cholesterol metabolism. Propionate is thought to suppress cholesterol synthesis, but some results have been inconclusive [6-10]. Acetate produced by the fermentation of oat bran may also contribute to cholesterol lowering [11].

Studying gut effects of fiber in humans is difficult due to the invasive and expensive nature of colonic observation as well as the dynamic nature of the colon. Excreted colon contents will not necessary represent colon contents in the proximal or even distal colon, due to continual fermentation of fiber and continual absorption of minerals and
SCFA across the epithelium. In vitro fermentation is a noninvasive, time-efficient means to estimate fiber fermentability.

The cholesterol lowering effect of whole oats has been well documented and the United States FDA issued a health claim on oats for the prevention of cardiovascular disease in 1996 [12]. However, limited research has been conducted with oat $\beta$-glucan separated from the oat grain. This current paper aims to determine if the beneficial physiological effects of oats can be seen with concentrated oat $\beta$-glucan.

A barrier to use of soluble fiber in the past has been the poor acceptability of foods high in soluble fiber for an extended period. We measured the effect of $6 \mathrm{~g} /$ day of concentrated $\beta$-glucan from oat on biomarkers of interest in CHD and CVD. The human feeding trial was designed to identify if the physiological effects of concentrated $\beta$ glucan from oat will improve serum biomarkers of cardiovascular risk in subjects at high risk of cardiovascular disease by measuring changes in total cholesterol, LDLcholesterol, HDL-cholesterol, triglycerides, apolipoprotein A-1, apolipoprotein B and other blood metabolites. Model intestinal fermentation was used to estimate in vivo short-chain fatty acid production.

\section{Methods}

Ninety hypercholesterolemic subjects were recruited for the study via flyers posted on the University of Minnesota campus and newspaper advertisements in the University of Minnesota Daily newspaper. To be included in the study, subjects needed to be healthy, non-smoking men and women between the ages of 22 and 65 years at risk for CVD (as defined by a total cholesterol greater than 200 $\mathrm{mg} / \mathrm{dl}$ ), with spoken and written English literacy. Subjects exclusion criteria included BMI > 30 upon admission to study; CVD, Diabetes Mellitus (fasting blood sugar $>126$ $\mathrm{mg} / \mathrm{dl}$ ), chronic inflammatory diseases (e.g. Crohn's, rheumatoid arthritis), cancer in prior 5 years, renal or hepatic disease, recent bacterial infection ( $<2$ weeks), acute febrile illness in prior 2 months, history of drug or alcohol abuse in prior 6 months, lipid-lowering, antihypertensive or anti-inflammatory steroid medication use, active weight loss $>5 \mathrm{~kg}$ in prior 3 months (intended or unintended), concurrent or recent (within 30 days) intervention study participation. The inclusion and exclusion criteria were assessed via telephone screening, a health history questionnaire, and the initial total cholesterol screening visit. Subjects received oral and written information about the study, including a comprehensive study newsletter and individual written consent was obtained from each subject. 
Subjects were asked to consume their usual diet with the addition of either a placebo supplement or dietary fiber from oat. The risks in the study were minimal. Subjects maintained their usual activities during the study with periodic visits for information sessions, sample collections, and supplement pickup.

\section{Study design}

The University of Minnesota Institutional Review Board Human Subjects Committee approved all aspects of this research. The study was a randomized, double-blind parallel group design. A total of 90 patients were enrolled with 45 patients per treatment arm. Subjects were randomly assigned to either placebo or treatment, stratified by age and sex. Fifteen subjects $(n=10$ treatment, $n=5$ placebo) were excluded from final analysis because their baseline cholesterol value was below $200 \mathrm{mg} / \mathrm{dl}$ despite a screening value above $200 \mathrm{mg} / \mathrm{dl}$. Treatment was 6 grams of concentrated $\beta$-glucan from oat per day (from $12 \mathrm{~g}$ oat bran concentrate containing $54 \%$ oat $\beta$ glucan). Placebo was 6 grams of dextrose monohydrate per day. Both were delivered as dietary supplements in the form of a powder. Subjects mixed their supplement with a beverage using a study-provided electric hand blender (Braun). Subjects were instructed to take the supplement via oral administration with their morning and evening meal for six weeks.

\section{Study visits}

Subjects attended an initial pre-screening visit to verify eligibility for the study (visit 1 ). Subjects who met study criteria were scheduled for a baseline visit, one day before the intervention began (visit 2). Visits 3 and 4 were scheduled for day 21 (midpoint) and day 42 (final) of the intervention.

\section{Diet records}

Three-day diet records were obtained from each subject at the midpoint and final visits. Subjects recorded two weekdays and one weekend day of their total food intake. All records were analyzed for total nutrient composition of each day's intake using the First DataBank Nutritionist Five software. Total calories, carbohydrate, fat, protein, cholesterol, saturated fat, monounsaturated fat, polyunsaturated fat, total fiber, soluble fiber, insoluble fiber and crude fiber were analyzed for each subject for average intake. Analysis of fiber did not include the addition of oat $\beta$-glucan to the diet.

\section{Blood samples}

At each visit subjects were asked to fast overnight for a minimum of 10 hours and then have blood samples drawn at the General Clinical Research Center, University of Minnesota, Twin Cities. Blood was drawn for the following measurements: plasma total cholesterol, triglycer- ides, HDL cholesterol, LDL cholesterol, apolipoprotein AI, apolipoprotein B, complete blood count and differential, homocysteine, c-reactive protein and comprehensive metabolic panel. Plasma LDL cholesterol concentration was calculated using the Friedewald equation. All specimens collected were transported via courier to Quest Diagnostics (Wood Dale, IL) for analysis.

\section{Anthropometric measurements}

Blood pressure was measured at each visit for each subject using a standardized sphygmomanometer (Tycos). Three measurements were taken, one minute apart, with the subject in a semi-reclined position following the approved National Institutes of Health method [13]. These three values were averaged individually for diastolic and systolic pressures. Standing weight was recorded every visit to the nearest $1 / 4 \mathrm{lb}$ with light clothing and no shoes on a calibrated balance beam scale.

\section{Symptom survey}

Each visit participants completed a short survey rating gastrointestinal effects on a scale related to normal. Symptoms recorded include: frequency of stools, consistency of stools, degree of intestinal bloating and degree of flatulence.

\section{Model intestinal fermentation}

Concentrated oat $\beta$-glucan (54\% $\beta$-glucan), partially hydrolyzed guar gum (MW $400 \mathrm{kDa})$, and inulin $(90 \% \mathrm{dp}$ $=10,10 \% \mathrm{dp}=1-2$ ) were subjected to model intestinal fermentation [14]. Glucose served as a positive control because it is fully fermentable by colonic bacteria. No fiber was the negative control to assess the concentrations of SCFA produced from substrates residing in the fecal inoculum. Chemical reagents were obtained from Fisher Scientific (New Hampton, NH, USA), Sigma Aldrich (St. Louis, MO, USA), and VWR Scientific (West Chester, PA, USA).

Briefly, the fibers were hydrated for 12 hours in $40 \mathrm{ml}$ sterile trypticase peptone media fortified with minerals, phosphate buffer, and a reducing solution $(950 \mathrm{ml}$ distilled water, $6.25 \mathrm{~g}$ cysteine hydrochloride, $40 \mathrm{ml} 1 \mathrm{~N} \mathrm{NaOH}$, $6.25 \mathrm{~g}$ sodium sulfide monohydrate) at $4^{\circ} \mathrm{C}$. Five- $100 \mathrm{~mL}$ serum bottles were prepared for each fiber sample $(0.5 \mathrm{~g})$, one for each of the five time points: $0,4,8,12$, and 24 hours. Control bottles containing either $0.5 \mathrm{~g}$ glucose or no added carbohydrate were prepared in the same manner. Two hours prior to inoculation with fecal solution, sample bottles were warmed to $37^{\circ} \mathrm{C}$. Fecal inoculum was prepared as described by McBurney and Thompson [14]. Fecal samples from three human subjects consuming a nonspecified Western diet were pooled (125 g total) and diluted with $400 \mathrm{ml}$ distilled water. The solution was 
homogenized in a blender. Reducing solution was added to the fecal ino 2 parts reducing solution [15].

Ten milliliters of fecal inoculum was added into each serum bottle along with $0.8 \mathrm{~mL}$ Oxyrase ${ }^{\circledast}$ oxygen reducing enzyme (Oxyrase Inc., Mansfield, $\mathrm{OH}$ ). The bottles were immediately flushed with carbon dioxide gas to eliminate oxygen and generate anaerobic conditions. The bottles were gently shaken in a $37^{\circ} \mathrm{C}$ water bath. One sample bottle for each fiber was removed at $0,4,8,12$, and 24 hours. Immediately upon removal, $1 \mathrm{~mL}$ of copper sulfate (200 $\mathrm{g} / \mathrm{L}$ ) was added to each bottle to kill the bacteria and cease fermentation. Two- $2 \mathrm{ml}$ aliquots were removed. Samples were prepared for gas chromatography as described previously $[16,17]$.

Lactate, acetate, propionate, butyrate isobutyrate, 2-methylbutyrate, isovalerate, and valerate were determined by gas chromatography using a Hewlett Packard 6890 gas chromatograph (Hewlett Packard, Palo Alto, CA) with a $4 \%$ carbowax $20 \mathrm{M} / 80 / 120$ carbopack B-DA column (Supleco, Bellefonte, PA) a temperature of 175 degrees C. Flow rates for nitrogen, hydrogen, and air were 24, 40, and $450 \mathrm{~mL} / \mathrm{min}$, respectively. All SCFA concentrations were corrected for the control concentration of SCFA at each time point. Molar ratios of SCFA were determined by dividing the number of moles of each SCFA (acetate, propionate, and butyrate) by the total moles of acetate, propionate, and butyrate.

\section{Statistical analysis}

Data was reported as means \pm SEM. Changes were calculated by subtracting baseline from the 6-week value, so that positive changes indicate an increase. Baseline values and changes were compared between oat $\beta$-glucan and control by two-sample t-tests, while paired t-tests were used for within-group changes from baseline. In the model intestinal fermentation, all SCFA concentrations were corrected for the negative control concentration of SCFA at each time point. SCFA concentrations were compared between fibers at each time point separately, using Tukey pairwise procedure for multiple comparisons. Statistical analyses were performed with SAS statistical software package, versions 8.0 and 9.1 (SAS Institute Inc, Cary, NC, USA).

\section{Results}

\section{Human trial}

Demographic and clinical characteristics of the oat $\beta$-glucan and control groups did not differ at baseline (Table 1). There were no changes from baseline in dietary intake, weight, or blood pressure in either group (Table 2). Total cholesterol significantly decreased within the treatment group ( $\mathrm{p}<0.025)$. Total cholesterol decreased $0.3 \pm 0.1$ $\mathrm{mmol} / \mathrm{L}$ (mean \pm SEM) in subjects consuming oat $\beta$-glu- can. Total cholesterol did not significantly change within the placebo group. Total cholesterol decreased $0.1 \pm 0.08$ $\mathrm{mmol} / \mathrm{L}$ (mean $\pm \mathrm{SEM}$ ) within the placebo group, but this change was not significant. Total cholesterol did not significantly change between groups.

LDL cholesterol significantly decreased within the treatment group ( $\mathrm{p}<0.025)$. LDL cholesterol dropped $0.3 \pm$ $0.1 \mathrm{mmol} / \mathrm{L}$ (mean \pm SEM) in subjects consuming oat $\beta$ glucan. LDL cholesterol did not change significantly within the placebo group; the mean decrease was 0.04 $\mathrm{mmol} / \mathrm{L}$. Treatment of oat $\beta$-glucan significantly lowered LDL cholesterol compared to control ( $\mathrm{p}<0.026)$. HDL cholesterol did not change significantly in either the treatment or placebo group.

Triglycerides increased $0.09 \pm 0.1 \mathrm{mmol} / \mathrm{L}($ mean \pm SEM) in the treatment group, but this change was not significantly different than 0 . Triglycerides fell $0.2 \pm 0.1 \mathrm{mmol} /$ $\mathrm{L}$ (mean $\pm \mathrm{SEM}$ ) in the placebo group. The triglyceride change between the treatment and control group was significantly different $(\mathrm{p}=0.030)$.

The oat $\beta$-glucan group experienced significant reduction from baseline in total cholesterol and LDL cholesterol, and the reduction in LDL cholesterol was greater than in the control group. Triglycerides were reduced in the control group but not the oat $\beta$-glucan group. Neither group showed changes in HDL cholesterol, C-reactive protein, homocysteine, insulin, or glucose.

Both groups reported no change in stool consistency, stool frequency, and bloating during the study, although the oat $\beta$-glucan group reported increased flatulence. Three-day food records completed on days 1-3, 22-24, and 39-41 were analyzed to verify dietary intake did not significantly change during the study. Changes in energy intake and diet composition are shown in Table 2.

\section{Model intestinal fermentation}

Concentrations of total short-chain fatty acids (TSCFA), acetate, propionate, and butyrate produced during the model intestinal fermentation are shown in Table 3. Concentrated oat $\beta$-glucan and inulin produced similar concentrations of TSCFA and were both significantly greater than guar gum at 4 hours. Oat $\beta$-glucan TSCFA production increased from 0 to 8 hours, and remained constant from 8 to 24 hours. Total short-chain fatty acid production by inulin peaked at 8 hours and decreased until 24 hours. Guar gum produced increasing concentrations of TSCFA at all time points. At 24 hours, guar gum produced the greatest concentrations of TSCFA while oat- $\beta$ glucan and inulin produced significantly few TSCFA. 
Table I: Baseline Demographic and Clinical Data

\begin{tabular}{|c|c|c|c|}
\hline & Treatment & Placebo & $P$ value \\
\hline n (female/male) & $35(22 / 13)$ & $40(28 / 12)$ & 0.513 \\
\hline Age (yr) & $44.5 \pm 2.2$ & $45.3 \pm 2.0$ & 0.796 \\
\hline $\begin{array}{l}\text { Blood pressure (systolic/diastolic, } \\
\mathrm{mmHg} \text { ) }\end{array}$ & $121 / 69 \pm 2.2 / 1.4$ & $121.6 / 67.1 \pm 1.9 / 1.5$ & $0.868 / 0.760$ \\
\hline Weight (kg) & $81.8 \pm 2.8$ & $79.6 \pm 2.9$ & 0.598 \\
\hline Total Cholesterol (mmol/L) & $6.2 \pm 0.1$ & $6.2 \pm 0.1$ & 0.976 \\
\hline Triglyceride $(\mathrm{mmol} / \mathrm{L})^{*}$ & $1.9 \pm 0.1$ & $1.9 \pm 0.2$ & 0.989 \\
\hline HDL cholesterol (mmol/L) & $1.4 \pm 0.06$ & $1.3 \pm 0.05$ & 0.806 \\
\hline LDL cholesterol (mmol/L) ${ }^{* *}$ & $4.1 \pm 0.1$ & $4.2 \pm 0.1$ & 0.651 \\
\hline Cholesterol/HDL ratio & $4.8 \pm 0.2$ & $4.8 \pm 0.2$ & 0.981 \\
\hline C-Reactive protein $(\mathrm{mg} / \mathrm{dL})$ & $0.35 \pm 0.08$ & $0.38 \pm .06$ & 0.817 \\
\hline Homocysteine $(\mu \mathrm{mol} / \mathrm{L})$ & $8.0 \pm 0.3$ & $7.9 \pm 0.3$ & 0.861 \\
\hline Insulin (pmol/L) & $56.2 \pm 4.2$ & $68.1 \pm 9.0$ & 0.273 \\
\hline Glucose (mmol/L) & $5.4 \pm 0.3$ & $5.3 \pm 0.1$ & 0.840 \\
\hline
\end{tabular}

Data are means \pm SEM.

* Excludes one subject in the Placebo group with fasting triglycerides $>400 \mathrm{mg} / \mathrm{dL}$.

*** Excludes two subjects in the Placebo group with fasting triglycerides $>400 \mathrm{mg} / \mathrm{dL}$

Acetate was the major contributor to TSCFA concentrations at all time points by all fibers. Acetate production mimicked the TSCFA production patterns with oat $\beta$-glucan and inulin being the fermented more rapidly than guar gum between 0 and 4 hours. At 8 hours, inulin produced significantly more acetate than oat $\beta$-glucan and guar gum. Acetate production at 12 hours was not different between the three fibers. At 24 hours, oat $\beta$-glucan produced the lowest concentrations of acetate, although this difference was only significant between guar gum and oat $\beta$ glucan.
Propionate was the SCFA produced in the second greatest concentration by oat $\beta$-glucan and guar gum. Oat $\beta$-glucan produced little propionate between 0 and 8 hours. From 8 to 24 hours, oat $\beta$-glucan produced propionate at a constant rate. Concentrations of propionate produced by oat $\beta$-glucan were less than guar gum but greater than inulin from 8 to 24 hours.

Oat- $\beta$ glucan produced the greatest butyrate concentrations from 4 to 12 hours. Between 12 and 24 hours, butyrate production by inulin increased dramatically, resulting in the greatest butyrate production at 24 hours. Oat $\beta$-glucan produced the second greatest concentration

Table 2: Comparison of endpoints at six weeks expressed as changes from baseline.

\begin{tabular}{|c|c|c|c|}
\hline & Treatment & Placebo & P value \\
\hline Total Cholesterol (mmol/L) & $-0.3 \pm 0.1 * * *$ & $-0.1 \pm 0.08$ & 0.184 \\
\hline LDL cholesterol $(\mathrm{mmol} / \mathrm{L})^{* *}$ & $-0.3 \pm 0.1 * * *$ & $-0.04 \pm 0.08$ & 0.026 \\
\hline HDL cholesterol (mmol/L) & $-0.02 \pm 0.02$ & $-0.009 \pm 0.02$ & 0.842 \\
\hline Triglyceride $(\mathrm{mmol} / \mathrm{L})^{*}$ & $0.09 \pm 0.1$ & $-0.2 \pm 0.1 * * *$ & 0.030 \\
\hline Cholesterol/HDL ratio & $-0.2 \pm 0.1$ & $-0.1 \pm 0.1$ & 0.947 \\
\hline C-Reactive protein (mg/dL) & $-0.03 \pm 0.06$ & $0.01 \pm 0.04$ & 0.546 \\
\hline Homocysteine $(\mu \mathrm{M} / \mathrm{L})$ & $0.4 \pm 0.4$ & $0.2 \pm 0.2$ & 0.528 \\
\hline Insulin (pmol/L) & $6.9 \pm 4.9$ & $4.2 \pm 10.4$ & 0.804 \\
\hline Glucose (mmol/L) & $0.2 \pm 0.09$ & $0.03 \pm 0.09$ & 0.259 \\
\hline Weight (kg) & $-0.7 \pm 0.3$ & $1.4 \pm 1.3$ & 0.117 \\
\hline $\begin{array}{l}\text { Blood pressure (systolic/diastolic, } \\
\mathrm{mmHg} \text {, value at end point) }\end{array}$ & $119 / 69 \pm 1.9 / 1.3$ & $119 / 69 \pm 2.0 / 1.7$ & $0.832 / 0.856$ \\
\hline Total Energy Intake per day (kJ) & $-519 \pm 330$ & $-343 \pm 360$ & 0.724 \\
\hline Carbohydrate intake (\% of total) & $0.4 \pm 2.0$ & $1.8 \pm 1.3$ & 0.535 \\
\hline Protein Intake (\% of total) & $0.9 \pm 0.6$ & $-0.9 \pm 1.1$ & 0.041 \\
\hline Fat Intake (\% of total) & $-0.7 \pm 1.8$ & $-1.2 \pm 1.1$ & 0.784 \\
\hline Dietary Fiber Intake (grams) & $-0.3 \pm 1.1$ & $-1.4 \pm 1.6$ & 0.454 \\
\hline
\end{tabular}

Data are means \pm SEM.

*** Statistically different from baseline, within group comparison $p<0.05$ 
Table 3: Total SCFA sample (MW $400 \mathrm{kDa}$ ) and inulin sample (degree of polymerization $100 \% \approx 10$ ) after $0,4,8,12$, and 24 hours in a model intestinal fermentation, analyzed by gas chromatography.

\begin{tabular}{|c|c|c|c|c|}
\hline Sample & & Oat $\beta$-glucan & Guar Gum & Inulin \\
\hline \multirow[t]{5}{*}{ Total SCFA } & $\mathrm{Oh}$ & $0.6 \pm 0.2$ & $-0.7 \pm 0.5$ & $-0.3 \pm 0.2$ \\
\hline & $4 \mathrm{~h}$ & $32.7 \pm 0.3 \mathrm{a} *$ & $7.7 \pm 0.02^{b}$ & $33.8 \pm 0.8^{a}$ \\
\hline & $8 \mathrm{~h}$ & $50.5 \pm 4.0^{a}$ & $45.7 \pm 3.2^{\mathrm{a}}$ & $75.4 \pm 0.7 b$ \\
\hline & $12 \mathrm{~h}$ & $50.3 \pm 6.2$ & $62.0 \pm 0.6$ & $55.6 \pm 1.8$ \\
\hline & $24 \mathrm{~h}$ & $48.7 \pm 1.5^{\mathrm{a}}$ & $68.6 \pm 0.8^{b}$ & $51.8 \pm 0.8^{a}$ \\
\hline \multirow[t]{5}{*}{ Acetate } & $\mathrm{Oh}$ & $-0.4 \pm 0.01$ & $-0.2 \pm 0.05$ & $-0.2 \pm 0.02$ \\
\hline & $4 \mathrm{~h}$ & $23.1 \pm 0.9 a$ & $6.6 \pm 0.5^{b}$ & $23.3 \pm 0.4^{a}$ \\
\hline & $8 \mathrm{~h}$ & $40.0 \pm 3.1^{a}$ & $34.2 \pm 2.2^{\mathrm{a}}$ & $63.7 \pm 1.8^{b}$ \\
\hline & $12 \mathrm{~h}$ & $37.5 \pm 4.5$ & $45.5 \pm 0.1$ & $48.2 \pm 4.2$ \\
\hline & $24 \mathrm{~h}$ & $24.7 \pm 1.7^{a}$ & $34.6 \pm 0.2^{b}$ & $30.3 \pm 0.8^{\mathrm{ab}}$ \\
\hline \multirow[t]{5}{*}{ Propionate } & $\mathrm{Oh}$ & $-0.1 \pm 0.01$ & $0.05 \pm 0.04$ & $-0.04 \pm 0.01$ \\
\hline & $4 \mathrm{~h}$ & $0.7 \pm 0.04$ & $0.5 \pm 0.1$ & $0.4 \pm 0.1$ \\
\hline & $8 \mathrm{~h}$ & $0.8 \pm 0.7 \mathrm{a}$ & $4.5 \pm 0.8^{b}$ & $-2.2 \pm 0.1^{a}$ \\
\hline & $12 \mathrm{~h}$ & $3.8 \pm 1.2^{\mathrm{a}}$ & $16.8 \pm 0.3^{b}$ & $-6.2 \pm 0.2^{c}$ \\
\hline & $24 \mathrm{~h}$ & $11.9 \pm 0.004^{a}$ & $28.5 \pm 0.7^{b}$ & $0.08 \pm 0.1^{c}$ \\
\hline \multirow[t]{5}{*}{ Butyrate } & $\mathrm{Oh}$ & $-0.1 \pm 0.02$ & $-0.03 \pm 0.04$ & $-0.03 \pm 0.02$ \\
\hline & $4 \mathrm{~h}$ & $0.4 \pm 0.02$ & $0.2 \pm 0.05$ & $0.2 \pm 0.04$ \\
\hline & $8 \mathrm{~h}$ & $2.2 \pm 0.3^{a}$ & $0.9 \pm 0.2^{b}$ & $0.6 \pm 0.05^{b}$ \\
\hline & $12 \mathrm{~h}$ & $7.7 \pm 0.9 a$ & $3.3 \pm 0.1 \mathrm{~b}$ & $-.004 \pm 0.1 \mathrm{c}$ \\
\hline & $24 \mathrm{~h}$ & $13.3 \pm 0.3^{\mathrm{a}}$ & $6.8 \pm 0.1^{b}$ & $23.1 \pm 0.1 c$ \\
\hline
\end{tabular}

† Total SCFA = acetate, propionate, butyrate, isobutyrate, 2-methylbutyrate, isovalerate, lactate, and valerate (if detectable).

$\ddagger$ Values shown are mean \pm standard error, each from 2 samples

* Mean production of each compound was compared at each time point between oat $\beta$-glucan, guar gum, and inulin. Within each row, means that do not share a superscript letter are significantly different $(p<0.05)$, and means that share a letter are not significantly different.

of butyrate at 24 hours, with guar gum producing the least butyrate.

\section{Discussion}

Large, prospective, epidemiologic studies find a protective effect of dietary fiber against coronary heart disease and form the basis for recommendations from the National Academy of Science for fiber intake (38 and 25 grams per day for young men and women)[18]. It has been proposed that a protective effect of soluble dietary fiber against CVD is mediated through direct or indirect effects on serum lipids. This study supports that oat $\beta$-glucan's role in CVD protection is through its effects on serum lipids. Virtually all other markers tested, other than serum lipids and apo B, did not significantly change as a result of 6 grams concentrated $\beta$-glucan.

Dietary fiber, as defined by the Institute of Medicine, consists of nondigestible carbohydrates and lignin that are intrinsic and intact in plants [19]. Functional fiber consists of concentrated, nondigestible carbohydrates that have beneficial physiological effects in humans [19]. The IOM recommended the elimination of the terms soluble and insoluble. New terms, viscous and fermentable, were recom- mended to describe the physicochemical properties of fibers. Viscous fibers such as $\beta$-glucan and guar gum have been shown to decrease blood LDL-cholesterol concentrations in animal models [20] and clinical intervention studies [21]. We have shown that consumption of oat $\beta$ glucan as a functional fiber has similar physiological effects as oat $\beta$-glucan intact in oats.

The reduction of cholesterol is possibly a sum of several effects. It is commonly accepted however that the majority of effect is due to decreased absorption of bile acids. This causes a removal of steroids from the body by fecal excretion resulting in increased catabolism of cholesterol, an increase in the secretion of bile acids, a decrease in lipoprotein cholesterol secretion, and a reduction in the total body pool of cholesterol [22]. Viscous fibers such as oat $\beta$ glucan can interfere with the absorption of dietary fat and cholesterol as well as enterohepatic recirculation of cholesterol and bile acids.

Viscous fibers may also delay the gastric emptying of ingested foods into the small intestine, resulting in a sensation of fullness, which may help weight control. Delayed gastric emptying may also reduce postprandial 
blood glucose concentrations leading to greater insulin sensitivity. In this study however, subjects consuming oat $\beta$-glucan or placebo had no change in weight. Perhaps a six week study is too short to impact subjects' weight. In addition, the oat $\beta$-glucan had no effect on fasting glucose or insulin concentrations.

Dietary fiber intake may also displace saturated fat intake and thus reduce CVD events [23]. However, three-day food records show no significant difference in saturated fat between treatment and placebo groups - supporting the idea that dietary fiber has beneficial cardiovascular effects independent of saturated fat.

The decrease in LDL cholesterol $(0.3 \mathrm{mmol} / \mathrm{L})$ as a result of oat $\beta$-glucan administration was a modest decrease. Statin treatment has been shown to decrease LDL cholesterol by $1.3 \mathrm{mmol} / \mathrm{L}$ over the course of a six week intervention [24]. However, the decrease in LDL cholesterol as a result of concentrated oat $\beta$-glucan administration is large enough to be clinically relevant. A $0.26 \mathrm{mmol} / \mathrm{L}$ increase in LDL cholesterol results in a $12 \%$ increase in risk of CVD [25].

Dietary interventions, such as The Portfolio diet which is high in plant sterols, soy protein, almonds, and viscous fiber, have been documented to reduce cholesterol levels as effectively as statin therapy [26]. The 12 gram dose of oat $\beta$-glucan used in this study was an acceptable dose to the subjects and provided clinically significant changes in cholesterol levels. The effectiveness of concentrated oat $\beta$ glucan as an add-on to statin therapy is beyond the scope of this study, but should be considered for future research. Concentrated oat $\beta$-glucan would be suitable as a standalone supplement to lower cholesterol. This product could also be used as a food ingredient to increase fiber content of food. When incorporated into minimally processed, low-fat food, concentrated oat $\beta$-glucan is hypothesized to retain is hypocholesterolemic effects.

This study also estimated the fermentability of concentrated oat $\beta$-glucan in a model intestinal fermentation system. Studying colonic fermentation in healthy humans is very difficult. The colonic epithelium is a dynamic system which is continually absorbing SCFAs as they are being produced by colonic bacteria. Currently, no method exists to measure SCFA absorption across the epithelium in humans. Short-chain fatty acid concentration in feces is a reasonable measurement of colonic fermentability. However, model intestinal fermentation is a more rapid, noninvasive, method of assessing fermentability and results from this method correspond with fermentability in vivo $[27,28]$.
Colonic fermentation varies between individuals as a result of species of gut bacteria and intestinal transit time. Absorption varies between individuals due to colonic retention time and other metabolites present in the feces, such as bicarbonate or sodium. The batch fermentation system eliminates differences in SCFA production due to the aforementioned variables and allows several fibers to be compared subjectively. Short-chain fatty acid production may play a role in decreasing cholesterol, but acetate and propionate concentrations were not remarkably different than concentrations produced by inulin or guar gum. Other mechanisms discussed above likely contribute to the cholesterol lowering feature of oat $\beta$-glucan.

The data from this study supports numerous studies showing increased intakes of viscous fiber decrease blood LDL-cholesterol concentrations [21]. Insulin concentrations, glucose concentrations, weight, homocysteine and CRP however did not significantly change in response to oat $\beta$-glucan and lead us to the question "Is this functional fiber different than dietary fiber?" It is likely that some food constituents, such as vitamins, trace elements, phenolic compounds, and phytoestrogens, found in fiber-rich foods also affect CVD risk and operate via pathways other than the lipid-regulating pathway [29]. The functional fiber, oat $\beta$-glucan, may be beneficial to subjects with high CVD risk because of its ability to significantly lower LDL cholesterol concentrations. However, several other mechanisms may underlie the cardiovascular benefits of dietary fiber. These include improvement in postprandial glucose and insulin responses and lowering of blood pressure and body weight [30]. Thus, the present study suggests that oat $\beta$-glucan significantly lowers LDL-cholesterol concentrations in subjects with high CVD risk.

\section{Conclusion}

A dosage of 6 grams concentrated $\beta$-glucan per day for six weeks produced significant reduction from baseline in total and LDL cholesterol. The reduction in LDL cholesterol was significantly greater than in the control group. These results indicate that concentrated $\beta$-glucan may be beneficial to high cardiovascular disease risk populations. Concentrated oat $\beta$-glucan is a fermentable fiber, with SCFA production similar to inulin and guar gum, as shown in model intestinal fermentation. This source of fiber will likely increase SCFA concentrations in the human colon, in addition to lowering LDL cholesterol.

\section{Competing interests}

The author(s) declare that they have no competing interests.

\section{Authors' contributions}

KMQ carried out the human study design, data review, and contributed to the manuscript. MLS carried out the 
model intestinal fermentation study, conducted statistical analysis, and contributed to the manuscript. KNS carried out the human study design and data review. WT served as the statistical advisor. GF participated as a co-principal investigator. JLS carried out the human study design and participated as a co-principal investigator.

\section{Acknowledgements}

Funding for this study was supported by MOI-RR00400, General Clinical Research Center Program, National Center for Research Resources, National Institutes of Health.

\section{References}

I. Lund EK, Johnson IT: Fermentable carbohydrate reaching the colon after ingestion of oats in humans. J Nutr 1991, | 21:3| |-3|7.

2. Lund EK, Gee JM, Brown JC, Wood PJ, Johnson IT: Effect of oat gum on the physical properties of the gastrointestinal contents and on the uptake of D-galactose and cholesterol by rat small intestine in vitro. Br J Nutr 1989, 62:91-101.

3. Marlett JA: Dietary fiber and cardiovascular disease. In Handbook of dietary fiber Edited by: Cho SS, Dreher ML. New York: Marcel Dekker; 2001:17-25.

4. Marlett JA, Hosig KB, Vollendorf NW, Shinnick FL, Haack VS, Story JA: Mechanism of serum cholesterol by oat bran. Hepatology 1994, 20:1450-1457.

5. Leiss O, vonBergmann K, Streicher U, Strotkoetler H: Effects of fibre components pectin, cellulose, and lignin on bile salt metabolism and biliary lipid composition in man. Gut 1986, 27:29-36.

6. Lin Y, Vonk RJ, Slooff MJ, Kuipers F, Smit MJ: Differences in propionate-induced inhibition of cholesterol and triacylglycerol synthesis between human and rat hepatocytes in primary culture. Br J Nutr 1995, 74: 197-207.

7. Wolever TM, Fernandes J, Rao AV: Serum acetate:Propionate ratio is related to serum cholesterol in men but not women. Nutr 1996, 126:2790-2797.

8. Wolever TM, Spadafora P, Eshuis H: Interaction between colonic acetate and propionate in humans. Am J Clin Nutr 1991, 53:68I-687.

9. Wolever TM, Spadafora PJ, Cunnane SC, Pencharz PB: Propionate inhibits incorporation of colonic $[1,2-13 \mathrm{C}]$ acetate into plasma lipids in humans. Am J Clin Nutr 1995, 6 I: I24I-1 247.

10. Wright RS, Anderson JW, Bridges SR: Propionate inhibits hepatocyte lipid synthesis. Proc Soc Exp Biol Med 1990, 195:26-29.

II. Bridges SR, Anderson JW, Deakins DA, Dillon DW, Wood CL: Oat bran increases serum acetate of hypercholesterolemic men. Am J Clin Nutr 1992, 56:455-459.

12. Food and Drug Administration, Human and Human Services: Food labeling: Health claims; oats and coronary heart disease: Proposed rule. Fed Regist 1996, 61:296-313.

13. National Institutes of Health (US) [NIH]: National High Blood Pressure Education Program. The Sixth Report of the Joint Nation Committee on Prevention, Detection, Evaluation, and Treatment of High Blood Pressure. Bethesda, MD 1997:98-4080.

14. McBurney MI, Thompson LU: Effect of human faecal inoculum on in vitro fermentation variables. BrJ Nutr 1987, 58:233-243.

15. Goering HK, Van Soest PJ: Forage fiber analysis: Apparatus, reagents, procedures, and some applications. In Agriculture Handbook No. 379 US Department of Agriculture. Washington, D. C.: US Government Printing Office; 1970: 1-20.

16. Pylkas AM, Juneja LR, Slavin JL: Comparison of different fibers for in vitro production of short chain fatty acids by intestinal microflora. J Med Foods 2005, 8: I I3-116.

17. Stewart M, Slavin JL: Molecular weight of guar gum affects short-chain fatty acid profile in model intestinal fermentation. Molecular Nutr Food Res 2006, 50:97I-976.

18. Lupton JR: Dietary fiber and coronary disease: Does the evidence support an association. Curr Atheroscler Rep 2003, 5:500-505.
19. Food Nutrition Board, Institute of Medicine: Dietary reference intakes: Proposed definition of dietary fiber. Edited by: Anonymous. Washington DC: National Academy Press; 2001.

20. Anderson JT: Dietary fiber, complex carbohydrate and coronary artery disease. Can J Cardiol 1995, II (supplement):55G-62G.

21. Glore SR, Van Treek D, Knehans AW, Guild M: Soluble fiber and serum lipids: A literature review. J Am Diet Assoc 1994, 94:425-436.

22. Malkki Y: Oat fiber. In Handbook of dietary fiber Edited by: Cho SS, Dreher ML. New York: Macrel Dekker; 200I:497-5I2.

23. Swain JL, Rouse IL, Curley C: Comparison of the effects of oat bran and low fiber wheat on serum lipoprotein levels and blood pressure. N Engl J Med 1990, 322:| $47-152$.

24. Cubeddu LX, Cubeddu RJ, Heimowitz T, Restrepo B, Lamas GA, Weinberg GB: Comparative lipid lowering effects of policosanol and atorvastatin: a randomized, parallel, double-blind, placebo-controlled trial. Am Heart J 2006, I52:el-5.

25. Howard BV, Robbins DC, Sievers ML, Lee ET, Rhoades D, Devereux RB, Cowan LD, Gray RS, Welty TK, Go OT, Howard W]: LDL cholesterol as a strong predictor of coronary heart disease in diabetic individuals with insulin resistance and low LDL: the strong heard study. Arterioscler Thromb Vasc Biol 2000:830-5.

26. Jenkins DJ, Kendall CW, Marchie A, Faulkner DA, Wong JM, de Souza R, Emam A, Parker TL, Vidgen E, Trautwein EA, Lapsley KG, Josse RG, Leiter LA, Singer W, Connelly PW: Direct comparison of a dietary portfolio of cholesterol-lowering foods with a statin in hypercholesterolemic participants. Am J Clin Nutr 2005, $81: 380-7$.

27. Wisker E, Daniel M, Rave G, Feldheim W: Short-chain fatty acids produced in vitro from fibre residues obtained from mixed diets containing different breads and in human faeces during the ingestion of the diets. Br J Nutr 2000, 84:3I-37.

28. Wisker E, Daniel M, Rave G, Feldheim W: Fermentation of nonstarch polysaccharides in mixed diets and single fibre sources: Comparative studies in human subjects and in vitro. BrJ Nutr 1998, 80:253-261.

29. Slavin JL, Martini MC, Jacobs DR Jr, Marquart L: Plausible mechanisms for the protectiveness of whole grains. Am J Clin Nutr 1999, 70(supplement):459S-463S.

30. Wu H, Dwyer KM, Fan Z, Shircore A, Dwyer JH: Dietary fiber and progression of atherosclerosis: The Los Angeles atherosclerosis study. Am J Clin Nutr 2003, 78: 1085- I091.

Publish with Bio Med Central and every scientist can read your work free of charge

"BioMed Central will be the most significant development for disseminating the results of biomedical research in our lifetime. "

Sir Paul Nurse, Cancer Research UK

Your research papers will be:

- available free of charge to the entire biomedical community

- peer reviewed and published immediately upon acceptance

- cited in PubMed and archived on PubMed Central

- yours - you keep the copyright

Submit your manuscript here:

http://www.biomedcentral.com/info/publishing_adv.asp
BioMedcentral 